\title{
Alterações promovidas pelo treinamento físico no edema pulmonar e perda de massa muscular em ratos portadores de tumor Walker-256
}

\section{Alterations caused by physical training in pulmonary edema and loss of muscle mass in rats with Walker-256 tumor}

\author{
Marli Cardoso Martins Pinge ${ }^{1}$; Rosiane Batista Mastelari²; \\ Igor Fernando Scanfelli da Silva ${ }^{3}$; Aida Mehanna ${ }^{4}$; \\ Samantha Bagolan de Abreu$^{5}$; Eleonora Elísia Abra Blanco ${ }^{6}$; \\ Helenir Medri de Souza ${ }^{7}$; Rubens Cecchini ${ }^{8}$
}

\begin{abstract}
Resumo
O tumor Walker-256 é um carcinoma de crescimento rápido e tem sido estudado sob vários aspectos metabólicos, associados ou não, à caquexia. Foi observado, em nosso laboratório, que em animais portadores de tumor Walker-256 após morte espontânea (geralmente em torno do décimo quinto dia) apresentavam edema pulmonar significativo com presença de líquido na cavidade pleural. Alguns trabalhos têm sugerido que o treinamento físico melhora a sobrevida de animais com tumor e minimiza os efeitos da caquexia. O objetivo de nosso trabalho foi o de avaliar o índice de edema pulmonar e massa muscular esquelética e cardíaca, além da sobrevida de ratos portadores de tumor Walker-256 submetidos previamente a treinamento físico por natação $(\mathrm{N})$. Para este estudo, foram usados ratos Wistar machos (200 a 220g), submetidos ao treinamento físico por natação (1 hora; 5 dias/semana, 4 semanas). Um dia após o treinamento, ratos sedentários $(\mathrm{C})$ ou treinados $(\mathrm{N})$ foram submetidos à inoculação no flanco direito de $8 \times 10^{7}$ células de tumor de Walker $256(\mathrm{~T})$. Imediatamente após a morte espontânea desses animais, foram avaliados o índice de edema pulmonar (IEP), a massa muscular esquelética (gastrocnêmio e soleus) e cardíaca. O edema pulmonar foi avaliado pelo índice calculado pela relação entre os pesos pulmonar e corporal de cada animal, e multiplicada por 100 (PP/PC x 100) (LEE et al., 2001). O índice de massa muscular (IMM) foi calculado de forma similar. Em animais normais, o IEP é igual a 0,53 $\pm 0,02(\mathrm{n}=20)$. Em ratos portadores de tumor após a morte espontânea apresentaram IEP significativamente maior $(2,62 \pm 0,31, \mathrm{n}=18)$. Após o treinamento físico em animais sem tumor, o IEP foi de $0,55 \pm 0,03(n=5)$. Já em animais portadores de tumor previamente treinados obteve-se um índice de edema inferior ao grupo controle com tumor $(1,46 \pm 0,16, n=5 ; p<0,05)$. Em relação à massa muscular, verificou-se, como esperado, uma diminuição no índice de massa muscular do gastrocnêmio no grupo controle com tumor, quando comparado ao grupo sem tumor $(\mathrm{C}=0,53 \pm 0,01 ; \mathrm{CT}=0,38 \pm$ $0,02)$. O mesmo ocorreu para o músculo soléus $(C=0,11 \pm 0,01 ; C T=0,08 \pm 0,01)$. O treinamento físico não modificou o IMM de todos os músculos avaliados quando comparados ao controle sem tumor. No entanto, no rato treinado com tumor, o IMM do músculo gastrocnêmio foi maior quando comparado ao
\end{abstract}

1 Profa. Dra. Docente do Departamento de Ciências Fisiológicas, Mestrado em Fisiologia pela UNICAMP, Doutorado em Farmacologia pela UNIFESP, Pós-doutorado na University of Columbia-Missouri, MO, USA. E-mail: martinspinge@uel.br.

2 Graduanda em Farmácia.

3 Graduando em Farmácia.

4 Mestre em Patologia Experimental.

5 Mestranda em Patologia Experimental.

6 Doutora em Fisiologia.

7 Doutora em Fisiologia.

8 Doutor em Bioquímica. 
grupo controle com tumor $(\mathrm{CT}=0,38 \pm 0,02 ; \mathrm{NT}=0,46 \pm 0,02, \mathrm{p}<0,05)$. Em relação ao músculo cardíaco, o treinamento físico não alterou o índice de massa cardíaca, mas a presença de tumor promoveu em ambos os grupos $(\mathrm{C}$ e $\mathrm{N})$ uma hipertrofia.Os nossos resultados mostram que o edema pulmonar está presente em animais portadores de tumor Walker 256 e que o treinamento físico parece atenuar o índice de edema nesses animais. O treinamento físico por si não modificou o IMM dos músculos avaliados, no entanto, a perda de massa pelo tumor no músculo gastrocnêmio foi menor no grupo treinado, e isso sugeriu que o exercício físico atenue a proteólise comumente observada em estados de caquexia e câncer.

Palavras-chave: Câncer. Exercício. Caquexia. Proteólise.

\section{Abstract}

Walker-256 tumor is a fast-growing tumor and has been studied under several metabolic aspects associated or not to cachexia. It was observed in our laboratory that animals with Walker-256 tumor, after spontaneous death (usually around the fifteenth day), showed significant pulmonary edema with fluid in the pleural cavity. Some studies have suggested that physical training improves the survival of animals with tumor and minimizes the effects of cachexia. The purpose of our work was to assess the pulmonary edema index as well as the cardiac and skeletal muscle mass, besides the survival of rats with Walker-256 tumor submitted previously to physical training through swimming $(\mathrm{N})$. For this study male Wistar rats (200 to $220 \mathrm{~g}$ ) were used, submitted to physical training through swimming (1 hour; 5 days a week, four weeks). One day after the training, sedentary rats $(\mathrm{C})$ or trained ones $(\mathrm{N})$ were submitted to inoculation on the right flank of $8 \times 10^{7}$ Walker-256 tumor cells (T). Immediately after spontaneous death of these animals, the pulmonary edema index (PEI), cardiac and skeletal muscle mass (gastrocnemius and soleus) were evaluated. Pulmonary edema was evaluated through the index calculated by the relation between lung and body weights of each animal, and multiplied by 100 (PP/PC $x$ 100) (LEE et al., 2001). Muscle mass (MM) index was calculated similarly. In normal animals the PEI is equal to $0,53 \pm 0,02(\mathrm{n}=20)$. In tumor-bearing rats after spontaneous death the PEI was significantly higher $(2,62 \pm 0,31, n=18)$. After the physical training in rats without tumor, the PEI was $0,55 \pm 0,03(n=5)$. Whereas in tumor-bearing rats previously trained, it was obtained a pulmonary edema index lower than that of the control group with tumor $(1,46 \pm 0,16, n=5 ; p<0,05)$. In terms of muscle mass, it was verified, as expected, a reduction in the gastrocnemius muscle mass index in the control group with tumor when compared to the group without tumor $(\mathrm{C}=0,53 \pm 0,01 ; \mathrm{CT}=0,38 \pm 0,02)$. The same thing happened for the soleus muscle $(\mathrm{C}=0,11 \pm 0,01 ; \mathrm{CT}=0,08 \pm 0,01)$. The physical training did not modify the MMI of all the muscles evaluated when compared to the control group without tumor. However, in the trained rat with tumor, the MMI of the gastrocnemius muscle was higher when compared to the control group with tumor $(\mathrm{CT}=0,38 \pm 0,02 ; \mathrm{NT}=0,46 \pm 0,02, \mathrm{p}<0,05)$. In terms of the cardiac muscle, the physical training did not alter the cardiac mass index, but the presence of the tumor caused in both groups $(\mathrm{C}$ and $\mathrm{N})$ a hypertrophy. Our results show that the pulmonary edema is present in animals with Walker-256 tumor and that the physical training seems to alleviate the edema index in these animals. The physical training itself did not modify the MMI of the evaluated muscles; however, the mass loss due to the tumor in the gastrocnemius muscle was lower in the trained group, suggesting that the physical exercise attenuated the proteolysis usually observed in cachexia and cancer conditions.

Keywords: Cancer.; Exercise.; Cachexia.; Proteolysis.

\section{Introdução}

Câncer é o termo comum utilizado para referirse a todos os tumores malignos. Os tumores tanto benignos quanto malignos, possuem dois componentes básicos:oparênquima, queéconstituído por células neoplásicas proliferantes, e o estroma de suporte, constituído de tecido conjuntivo e vasos sanguíneos (ROBBINS, 1991). O crescimento e a evolução das neoplasias dependem criticamente de seu estroma, pois ele irá determinar o suprimento sanguíneo adequado para o parênquima.

O crescimento de um tumor demanda energia, a qual deve ser provida do hospedeiro. Como conseqüência, há depleção das reservas de energia 
como do glicogênio no fígado e músculo, das proteínas musculares e dos triacilgliceróis do tecido adiposo. Dessa forma, é comum em portadores de câncer o desenvolvimento de caquexia, caracterizada por dramática perda de tecido muscular e adiposo, fraqueza, anorexia e anemia (FEARON, 1992).

O tumor de Walker-256 é um carcinoma de crescimento rápido, adequado para o estudo da caquexia. Em estudos com ratos, observa-se, dentro de um período de 14 dias do implante da massa tumoral, rápida perda de peso, seguida de morte ao final desse período (VICENTINO et al., 2002a, 2002b).

O edema pulmonar é uma patologia na qual há acúmulo anormal de líquido e de solutos nos tecidos extravasculares e/ou nos espaços aéreos do pulmão (COSTA; ANDRADE, 1995). O edema pulmonar ocorre em duas situações clínicas mais freqüentes: durante a falência ventricular esquerda, que compromete oretorno venoso da circulação pulmonar (edema pulmonar cardiogênico ou hemodinâmico), e durante a alteração de permeabilidade da membrana endotelial do capilar pulmonar, levando a um aumento do fluxo de proteínas do capilar para o espaço intersticial (edema pulmonar não cardiogênico) (KNOBEL, 1998). Essa alteração da permeabilidade pode ser causada por uma variedade de condições, como infecção pulmonar difusa, inalação de substâncias irritantes, sepse entre outras (COSTA; ANDRADE, 1995).

O exercício físico tem sido sugerido como um método preventivo e terapêutico no tratamento de várias condições patofisiológicas, tais como a doença cardiovascular e o câncer (COSTA ROSA, 2004). Estudos epidemiológicos fornecem evidências de que a prática de diferentes tipos de exercícios é capaz de influenciar a incidência de câncer, melhorar as condições de humanos e animais portadores de tumor, promover redução considerável nas taxas de mortalidade dos indivíduos, e serve, assim, como estratégia complementar, de baixo custo, para melhorar a qualidade de vida de pacientes com câncer e reduzir custos do sistema público de saúde (BACURAU et al., 2000; COSTA ROSA, 2004).

Em nosso laboratório, verificou-se que ratos portadores de tumor de Walker-256 com morte espontânea apresentavam edema pulmonar com extravasamento de líquido (resultados incluídos no grupo CT). Dessa forma, o objetivo deste trabalho foi avaliar o índice de edema pulmonar e massa muscular esquelética e cardíaca, de ratos portadores de tumor de Walker-256 submetidos previamente a treinamento físico pela natação.

\section{Metodologia}

\section{Animais}

Este trabalho foi realizado no Departamento de Ciências Fisiológicas (CIF) do Centro de Ciências Biológicas (CCB) da Universidade Estadual de Londrina (UEL) e todos os experimentos foram aprovados pelo Comitê de Ética em Experimentação Animal da Universidade.

Para esta pesquisa, foram utilizados 48 ratos Wistar machos, provenientes do Biotério Central da UEL. Os animais tiveram peso padronizado entre 200 e 220 gramas no início do treinamento, e foram mantidos em gaiolas coletivas (máximo de seis animais), à temperatura constante de $21 \pm 2{ }^{\circ} \mathrm{C}$, com ciclo claro/escuro de 12 horas. Água e ração foram fornecidas "ad libitum".

\section{Grupos experimentais}

Para a realização do presente estudo os animais utilizados foram divididos em 4 grupos experimentais:

- Controle (C): animais sedentários que receberam a inoculação de PBS no flanco direito

- Controle Tumor (CT): animais sedentários que receberam a inoculação de $8 \times 10^{7}$ células de tumor de Walker-256 no flanco direito. 
- Treinado (N): animais submetidos ao treinamento físico pela natação e que receberam a inoculação de PBS no flanco direito.

- Treinado Tumor (NT): animais submetidos ao treinamento físico pela natação e que receberam a inoculação de $8 \times 10^{7}$ células de tumor de Walker-256 no flanco direito.

\section{Treinamento fisico}

Os animais destinados ao treinamento físico tiveram seus pesos avaliados diariamente antes do início de cada sessão. O treinamento consistiu de 20 sessões (4 semanas) de uma hora de natação diária, durante cinco dias por semana, de acordo com Martins-Pinge et al. (2005).

$\mathrm{Na}$ primeira semana, houve aumento gradual do período de natação até alcançar o tempo de uma hora, iniciando com quinze minutos no primeiro dia, seguido de trinta minutos no segundo dia, quarenta e cinco minutos no terceiro dia e sessenta minutos a partir do quarto dia. Durante o período de treinamento, o grupo controle sedentário, teve seu peso avaliado paralelamente aos animais submetidos ao treinamento.

Os animais realizaram o treinamento em um tanque de vidro $(100 \times 60 \times 50 \mathrm{~cm})$ contendo água aquecida a $31 \pm 1^{\circ} \mathrm{C}$, a uma profundidade de 40 $\mathrm{cm}$. Após cada sessão de natação diária, os animais foram colocados em caixa para aquecimento e secagem. Depois de secos retornavam ao biotério.

Manutenção e Implantação do tumor de walker256

As etapas de manutenção e implantação do tumor Walker-256 foram realizadas segundo Vicentino e colaboradores, 2002b. O tumor Walker-256 foi cedido pelo laboratório de Metabolismo hepático da Universidade Estadual de Maringá (UEM). Ele era mantido através de passagens semanais por inoculação asséptica intraperitoneal de 2,0 x $10^{6}$ células/animal. Após 7 dias, o exudato peritoneal era retirado e submetido à centrifugação diferencial a $4^{\circ} \mathrm{C}$ para obtenção das células tumorais. As células eram suspensas em tampão fosfato $16,5 \mathrm{mM} ; 137$ $\mathrm{mM}$ de $\mathrm{NaCl}$ e 2,7 mM de $\mathrm{KCl}$ (PBS) gelado, com $\mathrm{pH}$ de 7,4. A viabilidade celular era avaliada pelo método de exclusão do Azul de Tripan em câmara de Neubauer.

Um dia após o treinamento físico, ratos sedentários ou treinados foram anestesiados com éter por um curto período de tempo e submetidos à inoculação no flanco direito de $8 \times 10^{7}$ células de tumor de Walker-256 (grupos CT e NT). Para os animais dos grupos $\mathrm{C}$ ou $\mathrm{N}$, foi realizado o mesmo procedimento, sendo inoculados com $500 \mu \mathrm{L}$ de PBS.

Cálculo do índice de edema pulmonar e massa muscular

Imediatamente após a morte espontânea, os animais foram submetidos à toracotomia após a qual os pulmões e o coração livres dos tecidos mediastinais foram retirados e pesados, para avaliação do índice de edema pulmonar (IEP). Além da retirada dos pulmões e coração, foi realizada também a retirada dos músculos sóleo e gastrocnêmio do membro posterior contralateral ao tumor, para análise dos pesos musculares e cálculo do índice de massa muscular (IMM). Esse índice é dado pela relação do peso muscular (PM) e peso corporal (PC) de cada animal multiplicado por 100 $(\mathrm{IMM}=\mathrm{PM} / \mathrm{PC}$ X 100).

O edema pulmonar foi avaliado pelo seu índice calculado pela relação entre os pesos pulmonar e corporal de cada animal e multiplicado por 100 (PP/ PC x 100) (LEE et al., 2001).

\section{Análise Estatística}

Os resultados foram expressos como média \pm erro padrão da média (EPM). O índice de edema 
pulmonar e o índice de massa muscular foram analisados inicialmente por Análise de Variância (ANOVA). As comparações aos pares entre sedentários e treinados, tumor e sem tumor, foram analisadas pelo teste t' de Student não pareado. As diferenças foram consideradas significantes para um valor de $\mathrm{p}<0,05$.

\section{Resultados}

Nos animais normais do grupo C o IEP foi igual a $0,53 \pm 0,02(n=20)$. Os ratos portadores de tumor de Walker-256 (CT), após a morte espontânea, apresentaram IEP significativamente maior $(2,62 \pm 0,31, n=18)$. Após o treinamento físico em animais sem tumor $(\mathrm{N})$, o IEP foi de $0,55 \pm 0,03$ $(n=5)$, que foi semelhante ao grupo C. Já nos animais portadores de tumor e previamente treinados (NT), estes apresentaram um índice de edema inferior ao grupo controle com tumor $(1,46 \pm 0,16, n=5 ; p<0,05)$ (Figura 1).

Em relação à massa muscular, verificou-se, como esperado, uma diminuição no índice de massa muscular do gastrocnêmio no grupo $\mathrm{CT}$, quando comparado ao grupo $\mathrm{C}(\mathrm{C}=0,53 \pm 0,01 ; \mathrm{CT}=0,38 \pm$ $0,02)$, e o mesmo ocorreu para o músculo soléus $(\mathrm{C}=0,11 \pm 0,01 ; \mathrm{CT}=0,08 \pm 0,01) . \quad \mathrm{O}$ treinamento físico não modificou o IMM de todos os músculos avaliados, quando comparados ao C. No entanto, no rato do grupo NT o IMM do músculo gastrocnêmio foi maior quando comparado ao grupo $\mathrm{CT}$ $(\mathrm{CT}=0,38 \pm 0,02 ; \quad \mathrm{NT}=0,46 \pm 0,02, \quad \mathrm{p}<0,05) . \quad \mathrm{Em}$ relação ao músculo cardíaco, o treinamento físico não alterou o índice de massa cardíaca, mas a presença de tumor promoveu em ambos os grupos (controle e treinado) um maior IMM (tabela 1).

Tabela 1. Valores médios do Índice de Massa Muscular (IMM) nos animais Controles e Treinados com e sem Tumor.

\begin{tabular}{ccccc}
\hline Músculo & $\begin{array}{c}\text { Controle } \\
(\mathrm{C})\end{array}$ & $\begin{array}{c}\text { Controle-Tumor } \\
(\mathrm{CT})\end{array}$ & $\begin{array}{c}\text { Treinado } \\
(\mathrm{N})\end{array}$ & $\begin{array}{c}\text { Treinado- Tumor } \\
(\mathrm{NT})\end{array}$ \\
\hline Sóleo & $0,11 \pm 0,01$ & $0,08 \pm 0,01$ & $0,11 \pm 0,01$ & $0,09 \pm 0,01$ \\
Gastrocnêmio & $0,53 \pm 0,01$ & $0,38 \pm 0,02 *$ & $0,56 \pm 0,03$ & $0,46 \pm 0,02^{\# \circ}$ \\
Cardíaco & $0,38 \pm 0,01$ & $0,51 \pm 0,03 *$ & $0,48 \pm 0,04^{* *}$ & $0,52 \pm 0,03$ \\
\hline
\end{tabular}

*Controle X Controle-Tumor $(\mathrm{p}<0,05) .{ }^{\circ}$ Treinado X Treinado-Tumor $(\mathrm{p}<0,05)$. \# Controle-Tumor X TreinadoTumor $(\mathrm{p}<0,05) .{ }^{* *}$ Controle $\mathrm{X}$ Treinado $(\mathrm{p}<0,05)$.

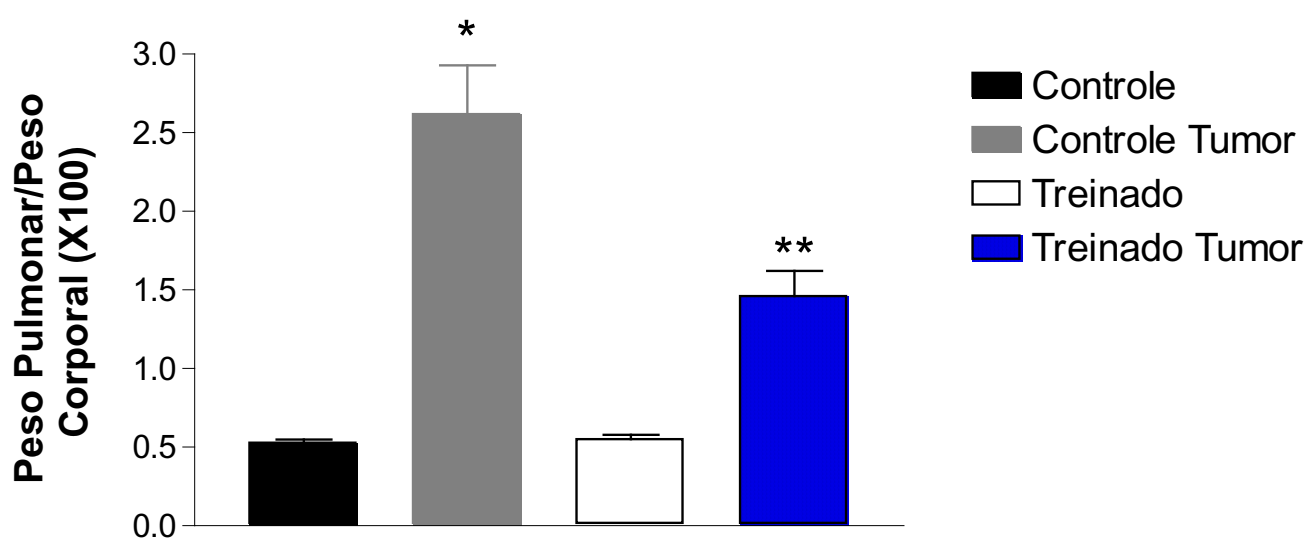

Figura 1. Índice de edema pulmonar nos vários grupos experimentais. * Diferente do grupo controle $(\mathrm{p}<0,05)$.

** Diferente do grupo controle tumor e grupo treinado $(\mathrm{p}<0,05)$. 


\section{Discussão}

Os nossos resultados mostraram que os animais portadores de tumor de Walker-256 apresentam na fase final da vida, edema pulmonar contendo um líquido turvo na cavidade torácica, rico em proteínas. Estes dados indicam que pelo menos parte deste edema possa ser de origem inflamatória. Sem dúvida, seguindo-se ao implante do tumor, ocorre uma ativação da resposta imune com o aumento de citocinas pró-inflamatórias tais como, TNF $\alpha$, IL1 e IL6 (TAYEK; \& BRASEL, 1990; ARGILÉS; \& LÓPES-SORIANO, 1990; SIDDIQUI; \& WILLIAMS, 1990). Uma vez instalado o edema inflamatório primário, ocorre um aumento da resistência pulmonar com subsequente hipertrofia do coração direito ("Cor pulmonale") e diminuição do volume minuto cardíaco por deficiente enchimento do ventrículo esquerdo. Este quadro levaria a uma amplificação do edema pulmonar secundário ao edema inflamatório primário (ROBBINS, 1991). Tal fato pode estar ocorrendo em nosso estudo o qual mostra hipertrofia cardíaca nos animais portadores do tumor. Adicionalmente, a significativa, porém parcial, redução do edema, provocada pelo treinamento, favorece a interpretação da existência de um evento hemodinâmico associado ao edema. A melhor capacidade de ejeção tanto do ventrículo direito como do esquerdo, produzida pelo exercício e não a hipertrofia, poderia estar na base da redução do componente hemodinâmico do edema. Esta afirmação é suportada pela nossa observação de que o treinamento não produziu adicional hipertrofia cardíaca nos animais com tumor, quando comparado com os animais sedentários portadores de tumor. Todavia, o treinamento físico de animais normais, promoveu aumento de massa muscular cardíaca, possivelmente por outros mecanismos. Esse resultado está de acordo com outros da literatura que mostram que o treinamento físico pela natação em ratos promove aumento de 12 a $31 \%$ no índice de massa muscular cardíaca (SCHAIBLE et al.; SCHEUER, 1979; EVANGELISTA; BRUN; KRIEGER et al., 2003).
O interesse no exercício físico como meio para a prevenção e proteção do câncer vem aumentando significativamente (COSTA ROSA, 2004). O efeito de um programa de atividade física, na redução da fadiga relacionada ao câncer e melhora na qualidade de vida, mostra-se positivo em pacientes em tratamento contra o câncer, fornecendo uma ferramenta adicional aos efeitos fisiológicos e psicossociais para o paciente com câncer (LOSITO; MURPHY; THOMAS, 2006).

Evidências epidemiológicas têm sugerido que a prática de atividade física regular, com intensidade moderada a intensa, reduz a incidência de câncer de colo e de mama. Em relação aos cânceres de próstata, pulmão e endométrio as evidências são menores (KRUG; ABOUL-ENEIN, 2006).

Em experimentos com animais os benefícios do exercício físico se mostram eficazes quando a realização deste ocorre no início da implantação do tumor ou nas fases promotoras. Estudos de Macneil e Hoffman-Goetz (1993) sugerem que o exercício poderia aumentar a função natural imune citotóxica por até três semanas após a cessação da atividade. Entretanto, este aumento na função imune não foi associado com redução de incidência de tumor nos animais exercitados.

Nossos dados também mostraram que animais previamente treinados não apresentavam um tempo de sobrevida maior, embora, apresentassem um índice de edema pulmonar menor. Provavelmente, outros fatores colaboraram para a morte do animal, já que vários mecanismos estão alterados nos animais portadores de tumor.

Em relação ao IMM do gastrocnêmio, observamos que os animais controle portadores de tumor apresentaram um índice menor quando comparado ao grupo controle sem tumor, caracterizando o quadro de perda de massa muscular. Os animais do grupo treinado não tiveram alteração do IMM, quando comparado com os animais do grupo controle. No entanto, os animais treinados com tumor apresentaram um IMM maior comparado ao grupo 
controle com tumor. Essa menor perda de massa muscular pelo treinamento físico pode ser resultado de uma inibição de processos bioquímicos ativados pelo tumor que induzem a proteólise. É conhecido que a proteólise muscular envolve vias lisossomais e não-lisossomais, e a proteólise não-lisossomal é controlada pelo sistema ubiquitina-proteassoma e involve a quebra de proteínas intracelulares e miofibrilares (KEE at al., 2002). A via da ubiquitina muscular é um processo complexo ativadado durante catabolismo severo, como aquele que ocorre durante a caquexia do câncer (ATTAIX et al., 1999; WRAY; MAMMEN; HASSELGREN, 2002; VENTRUCCI; MELLO; GOMES-MARCONDES, 2004).

\section{Conclusões}

Os nossos resultados mostraram que o edema pulmonar está presente em animais portadores de tumor de Walker 256. Aparentemente, este edema é formado por dois eventos: inflamatório e hemodinâmico e que o treinamento físico parece atenuar o índice de edema nesses animais por atuar principalmente no evento hemodinâmico. $\mathrm{O}$ treinamento físico, por si, não modificou o IMM dos músculos avaliados, no entanto a perda de massa pelo tumor no músculo gastrocnêmio foi menor no grupo treinado, sugerindo que o exercício físico atenue a proteólise comumente observada em estados de caquexia e câncer.

\section{Referências}

ARGILÉS, J. M.; LÓPEZ-SORIANO, F. J. The effects of tumour necrosis factor-alpha (cachectin) and tumour growth on hepatic amino acid utilization in the rat. The Biochemical Journal, London, v. 266, n. 1, p. 123-126, 1990.

ATTAIX, D.; COMBARET, L.; TILIGNAE, T.; TAILLANDIER, D. Adaptation of the ubiquitinproteasome proteolytic pathway in cancer cachexia. Molecular Biology Reports, Dordrecht, v. 26, n. 1-2, p. 77-82, 1999.
BACURAU, R. F. P.; BELMONTE, M. A.; SEELAENDER, M. C.; COSTA ROSA, L. F. Effect of moderate intensity exercise training protocol on the metabolism of macrophages and lymphocytes of tumorbearing rats. Cell Biochemistry and Function, Chichester, v. 18 , n. 4, p. 249-258, 2000.

COSTA ROSA, L. F. B. P. Exercise as a time-conditioning effector in chronic disease: a complementary treatment strategy. Evidence Based Complement Alternative Medicine, Oxford, v., 1, n. 1, p. 63-70, 2004.

COSTA, A. F; ANDRADE, G. N. Edema pulmonar. In: BETHLEN, N. Pneumologia. 4. ed. São Paulo: Atheneu, 1995. p. 811-816.

EVANGELISTA, F. S.; BRUN, P. C.; KRIEGER, J. E. Duration-controlled swimming exercise training induces cardiac hypertrophy in mice. Brazilian Journal of Medical and Biological Research, Ribeirão Preto, v. 36, n. 12, p.1751-1759, 2003.

FEARON, K. C. H. The mechanisms and treatment of weight loss in cancer. Proceedings of the Nutrition Society, Wallingford, v. 51, n. 22, p. 251-260, 1992.

KEE, A. L.; COMBARET, L.; TILIGNAE, T.; SOUWEINE, B.; AUROUSSEAU, E.; DALLE, M.; TAILLANDIER, D.; ATTAIX, D. Ubiquitin-proteasomedependent muscle proteolysis responds slowly to insulin release and refeeding in starved rats. Journal of Physiology, Paris, v. 546, n. 3, p. 765-776, 2002.

KNOBEL, E. Edema Agudo de Pulmão. In: (Org.). Condutas no Paciente Grave. 2. ed. São Paulo: Atheneu, 1998. p. 177-185.

KRUG, J.; ABOUL-ENEIN, H. Y. Physical activity in the prevention of cancer. Asian Pacific Journal of Cancer Prevention, Bangkok, v. 7, n. 1, p. 11-21, 2006.

LEE, R. P., WANG, D., KAO, S. J., CHEN, H. I. The lung is the major site that produces nitric oxide to induce acute pulmonary oedema in endotoxin shock. Clinical and Experimental Pharmacology and Physiology, Oxford, v. 28 , n. 4 , p. $315-320,2001$

LOSITO, J.; MURPHY, S.; THOMAS, M. The effects of group exercise on fatigue and quality of life during cancer treatment. Oncology Nursing Forum, Pittsburgh, v. 33, n. 4, p. 821-825, 2006.

MAcNEIL, B.; HOFFMAN-GOETZ, L. Effect of exercise on natural cytotoxicity and pulmonary tumor metastases in mice. Medicine and Science in Sports and Exercise, Hagerstown, v. 25, n. 8, p. 922-928, 1993. 
MARTINS-PINGE, M. C.; BECKER, L. K.; GARCIA, M. R. L.; ZOCCAL, D. B.; NETO, R. V.; BASSO, L. S.; SOUZA, H. C.; LOPES, O. U. Attenuated pressor responses to amino acids in the rostral ventrolateral medulla after swimming training in conscious rats. Autonomic Neuroscience, Amsterdam, v. 122, n. 1-2, p. 21-28, 2005.

ROBBINS, S. L. Neoplasia. In: Patologia estrutural e funcional. 4. ed. Rio de janeiro: Guanabara Koogan, 1991. p. 267-291.

SCHAIBLE, T. F.; SCHEUER, J. Effects of physical training by running or swimming on ventricular performance of rat hearts. Journal of Applied Physiology, Bethesda, v. 65, n. 4, p. 116-123, 1979.

SIDDIQUI, R. A.; WILLIAMS, J. F. Tentative identification of the toxohormones of cancer cachexia: roles of vasopressin, prostaglandin E2 and cachectinTNF. Biochemistry international, Marrickville, v. 20, n. 4, p. 787-797, 1990.

TAYEK, J. A.; BRASEL, J. A. Effects of tumor necrosis factor alpha on skeletal muscle and Walker 256 carcinosarcoma protein metabolism studied in vivo. Cancer Research, Baltimore, v. 50, n. 9, p. 2765-2768, 1990.
VENTRUCCI, G.; MELLO, M. A. R.; GOMESMARCONDES, M. C. C. Proteasome activity is altered in skeletal muscle tissue of tumor-bearing rats fed a leucine-rich diet. Endocrine-Related Cancer, Woodlands, v. 11, n. 4, p. 887-895, 2004.

VICENTINO, C.; CONSTANTIN, J.; BRACHT, A.; YAMAMOTO, N. S. Long-chain fatty acid uptake and oxidation in the perfused liver of Walker-256 tumorbearing rats. Liver, Copenhagen, v. 22, n. 4, p. 342-350, 2002 a.

VICENTINO, C.; CONSTANTIN, J.; STECANELLA, L. A.; BRACHT, A.; YAMAMOTO, N. S. Glucose and glycogen catabolism in perfused livers of Walker256 tumor-bearing rats and the response to hormones. Pathophysiology, New York, v. 8, n. 3, p. 175-182, Jun. 2002b.

WRAY, C. J.; MAMMEN, J. M.; HASSELGREN, P. O. Catabolic response to stress and potential benefits of nutrition support. Nutrition, London, v. 18, n. 11, p. 971977, Nov. 2002. 